\title{
Comparative study of two elasto-plastic work- hardening spatial models for concrete
}

\author{
Pawet M. Lewiński ${ }^{1, *}$, and Marta Zygowska ${ }^{2}$ \\ ${ }^{1}$ Building Research Institute (ITB), Filtrowa 1, 00-611 Warszawa, Poland \\ ${ }^{2}$ Ramboll Polska Sp. z o. o., Młynarska 48, 01-171 Warszawa, Poland
}

\begin{abstract}
A concept of elasto-plastic, work-hardening constitutive models for the multiaxial behaviour of concrete under short-term loading and the comparison with test results is presented in this paper. Two failure surfaces are utilized: the criterion of Podgórski and the three-parameter surface of Willam and Warnke. Both triaxial failure criteria have been calibrated in terms of different multiaxial strength tests. A non-associated flow rule has been used. The plastic potential function has been assumed in the form of the Drucker-Prager cone with variation of the angle of the cone side surface. In order to cover the plastic hardening behaviour, the equivalent uniaxial stress-strain curve has been adopted. An incremental stress-strain relationship has been formulated. The results of the numerical analysis performed by a direct integration of the constitutive relationships for the biaxial stress regime have been compared with the test data.
\end{abstract}

\section{Introduction}

Concrete is considered as the most widely used construction material. With regard to the assessment of its mechanical properties, one of the main factors is the knowledge about the strength properties of the material, where the main role is played by the analysis of the structural behaviour in the spatial stress state. The modelling of the mechanical properties of concrete has become possible through the advances in research technology as well as computer and numerical methods in recent years. Papers by many researchers provided detailed data on the physical properties of concrete in complex stress states. The main reason for discrepancies between the results is probably the method of transferring the load onto the specimen. This can be done using the "steel brushes" to eliminate friction, as described in the works of Kupfer et al. [1,2], or by means of rigid steel platens (see, for example, Schickert and Winkler [3]). A more recent paper by Zisopoulos, Kotsovos and Pavlovic [4] on the behaviour of concrete in compression under different boundary conditions confirms that plain concrete can be described as a brittle material. An essential element in the analysis of the behaviour of concrete occupying researchers over the years has been the shape of the failure surface in the stress space. The experimental results for brittle materials indicate that the shape of the failure surface depends significantly on the first stress tensor invariant and both on the second and the third invariants of stress tensor

\footnotetext{
* Corresponding author: p.lewinski@itb.pl
} 
deviator. Most often it is assumed that the failure surface is open in the direction of the hydrostatic compressive stress. The surface has curved meridians and the deviatoric crosssections in the form of closed envelope changing from an almost triangular shape for low stress level to the circular one for high compressive stresses. These conditions have been taken into account in the works of many researchers, including Willam and Wranke [5], A.C.T. Chen and W.F. Chen [6], Ottosen [7], Lade [8], Podgórski [9], Ohtani and Chen [10]. The five-parameter model of Willam et al. $[5,11]$ was initially considered to be quite complicated, but since the elliptic function $r(\square, e)$ (mentioned below) was developed by Klisiński [12], it has proved to be very effective. Similarly, in the case of the failure surface of Podgórski [9], some simplifications (see below) were proposed by Lewiński [13] (without the loss of accuracy). An interesting concept of the failure surface was presented in the paper by Jemiol o and Szwed [14], with the failure function involving 3 mutually orthogonal invariants of stress state. The elastic-plastic model of concrete with non-linear hardening, based on a non-associated flow rule enables the correct description of the phenomenon of dilatancy, i.e. the increase in the volume of concrete as a result of the formation of microcracks for the compressive stresses close to the failure. To obtain such description of the dilatancy phenomenon, it is necessary to develop the appropriate formulation of the plastic potential function and such a concept is a subject of this paper. A stepwise departure from associativity, described by the membership function was proposed by Jemiol o et al. [15]. In the present model the nonlinear properties of concrete are described in a similar way as presented at AMCM 2011 conference by Lewiński and Zygowska [16], however, some improvements have been introduced in the description of the failure surface and its evolution. A comprehensive review of the state of the art in the plasticity modelling of the mechanical behaviour of plain concrete together with its applications in reinforced concrete structures, including the numerical implementation, was given by Chen $[17,18]$. W. F. Chen in his monographic book [18] wrote: "The latest step in the development of concrete constitutive models and finite-element reinforced concrete applications is the work-hardening theory of plasticity". The application of proposed models enables the correct and test-based description of the dilatancy phenomenon via effective control of the shape of the plastic potential function evolution. Thus, the general aim of the paper is to devise the models which are relatively simple and convenient for using in the numerical analysis of concrete structures and moreover are able to serve as a starting point to devise a more sophisticated model, e.g. continuum damage model [19] based on failure criteria for concrete described herein.

\section{Failure surfaces}

In case of total strain constitutive model for concrete the ultimate surface proposed by $\mathrm{J}$. Podgórski (JP) [9] was implemented. In the failure criterion assumed herein, the basic parameters can be written in the following manner (see Figure 1):

$$
r(\varphi)=\sqrt{2 J_{2}}=[P(J)]^{-1}, P(J)=\cos [(\arccos (\alpha J)) / 3-\beta], J(\varphi)=\cos 3 \varphi=\frac{3 \sqrt{3} J_{3}}{2 J_{2}^{3 / 2}},
$$

where $\alpha$ and $\beta$ are constants fulfilling the following inequalities: $0 \leq \alpha \leq 1$ and $0 \leq \beta \leq$ $\pi / 6$.

The parameters describing the proportion of radius $r$ for different $\varphi$ angles are as follows:

$$
\lambda=r\left(\varphi=0^{\circ}\right) / r\left(\varphi=60^{\circ}\right) \quad \vartheta=r\left(\varphi=30^{\circ}\right) / r\left(\varphi=60^{\circ}\right) .
$$


According to Podgórski [9], $\alpha$ and $\beta$ coefficients from equation (1) can be derived by means of iteration. However, the equations for the direct calculation of these parameters were proposed by Lewiński [13]. From the set of equations (1) and (2) for $\varphi=0^{\circ}, 30^{\circ}, 60^{\circ}$ the trigonometric equations can be obtained which can be solved with regards to $\alpha$ and $\beta$ :

$$
\alpha=\sin 3 \alpha_{0}, \beta=\frac{\pi}{6}-\arctan \left(\frac{\vartheta(1-\lambda)}{2 \lambda \sin \alpha_{0}}\right), \quad \text { where } \alpha_{0}=\arccos \left(\frac{\vartheta}{2}\left(1+\frac{1}{\lambda}\right)\right) .
$$

J. Podgórski confirmed the usefulness of the above expressions [20, 21]. Assuming that in the octahedral set of coordinates the meridian of ultimate surface is described by a parabolic equation, the following equation for the failure surface was used (see Figure 2):

$$
F\left(\sigma_{o c t}, \tau_{o c t}, \varphi\right)=\sigma_{o}-C_{0}+C_{1} P(J) \tau_{o}+C_{2} \tau_{o}^{2}=0
$$

The values of $C_{0}, C_{1}, C_{2}$ can be derived on the basis of experimental data in the following way:

$$
C_{0}=f_{t}, C_{1}=\frac{\sqrt{2}}{P(\varphi=0)}\left[1-\frac{3}{2} \frac{\left(f_{t} / f_{c c}\right)}{\left(f_{c c} / f_{t}-1\right)}\right], C_{2}=\frac{9}{2} \frac{f_{t} / f_{c c}}{f_{c c}-f_{t}}
$$

Such defined 5-parameter failure surface for concrete poses no problem with numerical implementation and complies with experimental results. The well-known criterion proposed by Willam, Menétrey and Warnke (W\&W) $[5,11]$ for the meridional crosssections takes the form:

$$
F(\xi, \rho, \theta)=A_{f}^{2} \rho^{2}+m\left[B_{f} \rho r(\theta, e)+C_{f} \xi\right]-c=0 .
$$

where the constants $A f, B_{f}, C_{f}$ and $m$ are expressed by the strength parameters of concrete. A shape of the deviatoric cross-section is made up of three symmetrical cuts of the ellipse and is described by the function $r(\theta, e)$ in the Haigh-Westergard cylindrical system $[11,12]$.

\section{Constitutive models for concrete}

The concept of the constitutive models for concrete under compression is based on the conventional theory of plastic flow with the emphasis on energy-type hardening. Such a model of plastic flow with nonlinear hardening can be interpreted as a model utilizing the infinite number of different surfaces in the mixed (isotropic, kinematic and anisotropic) hardening rule (Eqs. (7)-(11)). In the studied models, the energy consistent effective stresses $\sigma_{0}$ and strains $\varepsilon p$ have been introduced. The non-linear hardening law is based on the Madrid Parabola formulation as follows:

$$
\sigma_{0}=\frac{2 f_{c}\left(1-\alpha_{0}\right)}{\varepsilon_{0 p}}\left(1-\frac{\varepsilon-\varepsilon_{s}^{*}}{2 \varepsilon_{0 p}}\right)\left(\varepsilon-\varepsilon_{s}^{*}\right)+\alpha_{0} f_{c}
$$

where: $\varepsilon=\varepsilon e+\varepsilon p$ and:

$$
\varepsilon_{e}=\frac{\sigma_{0}}{E_{0}}, \varepsilon_{s}^{*}=\frac{\alpha_{0} f_{c}}{E_{0}}, \varepsilon_{0 p}=\frac{2 f_{c}\left(1-\alpha_{0}\right)}{E_{0}}
$$

where: $E 0$ - Young's modulus, $\alpha_{0}$ - the parameter of the end of elastic range. 
The effective value of energy consistent strain has been introduced in order to obtain better compliance with the experimental results:

$$
\varepsilon_{0 p}^{*}=\psi \frac{2 f_{c}\left(1-\alpha_{0}\right)}{E_{0}}=\psi \varepsilon_{0 p},
$$

where the parabolic variation of the coefficient $\psi$ depending on the relations of the principal stresses $\zeta_{1}=\sigma_{2} / \sigma_{1}$ and $\zeta_{2}=\sigma_{3} / \sigma_{1}$ can be expressed as follows:

$$
\psi=1,5\left[1+\left(2-1,4 \zeta_{1}\right) \zeta_{1}\right] \cdot\left[1+\left(2-1,4 \zeta_{2}\right) \zeta_{2}\right] \text {. }
$$

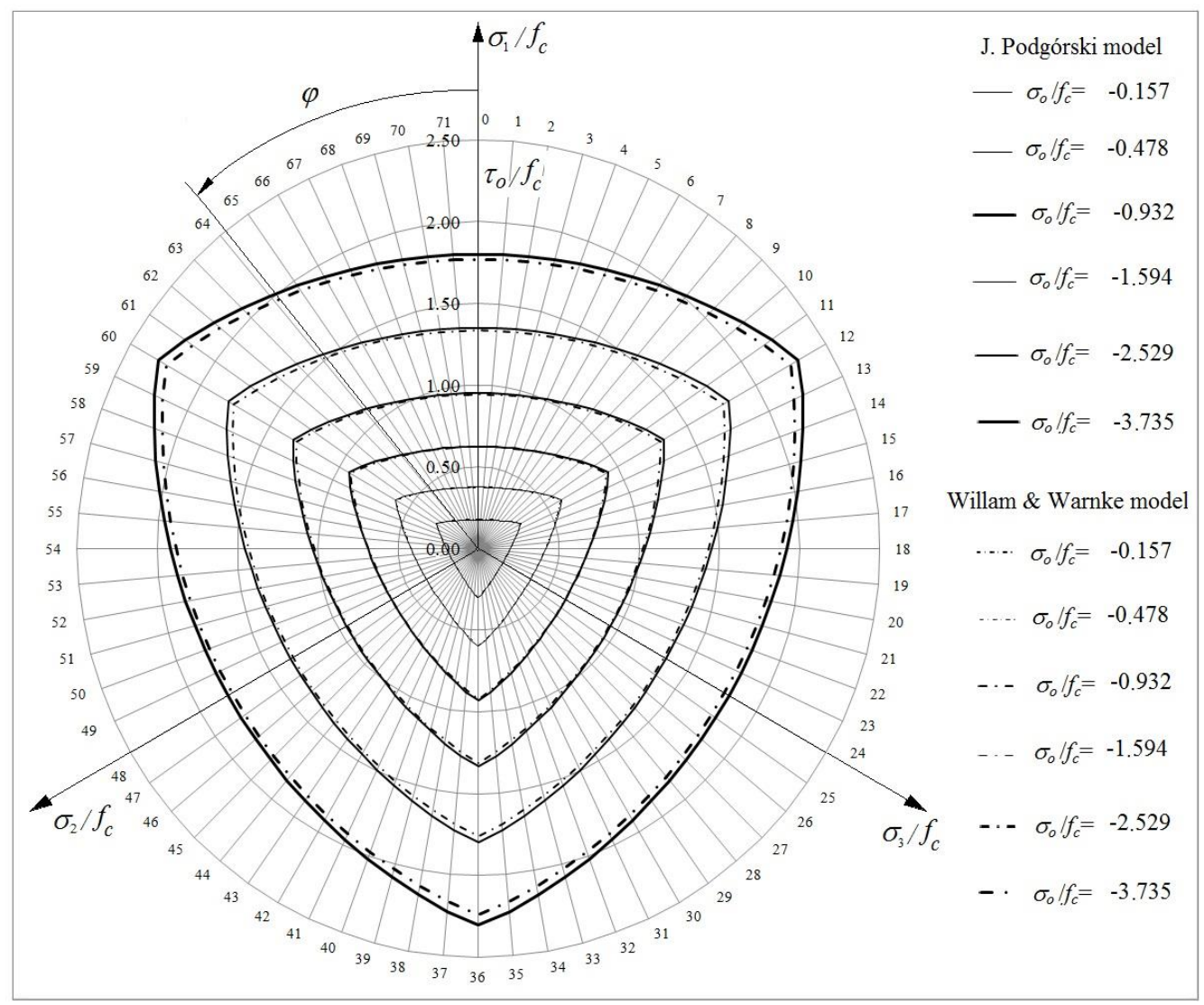

Fig. 1. Comparison of JP [9] and W\&W [1,2] failure surfaces for the cross-sections by the deviatoric planes (for different values of $\sigma_{o} / f_{c}$ ) in the case of $e=0.514$, peripheral coordinates pts. - every $5^{\circ}$. 


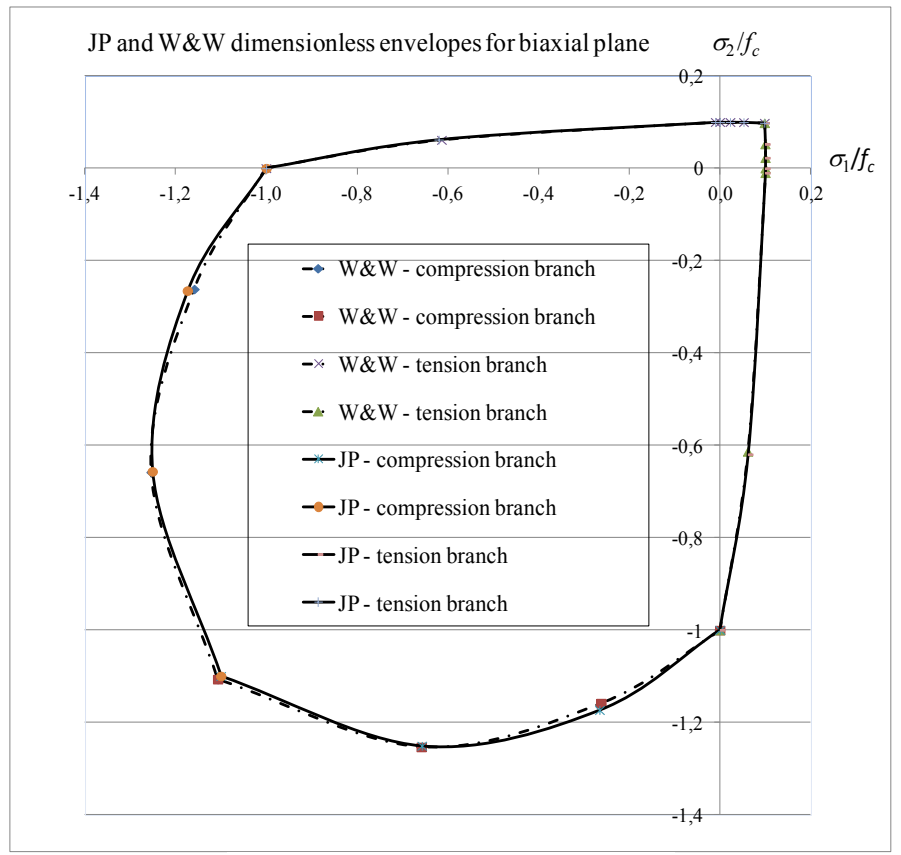

a) Comparison for plane stress state

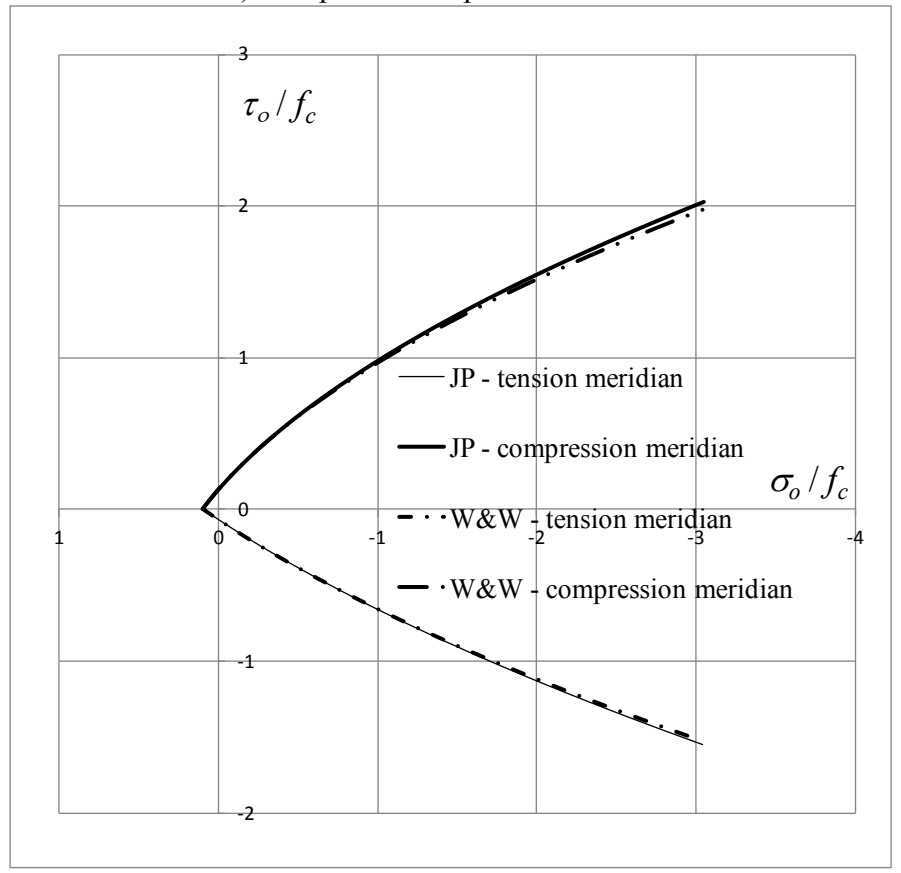

b) Comparison for meridional cross-sections

Fig. 2. Comparison of the cross-sections of failure surfaces: JP [9] and W\&W [1, 2] for $e=0.514$.

An introduction of the coefficient $\psi$ was aimed to calculate actual values of strain at maximum stress, yield in tests by Kupfer et al. $[1,2]$, as it is a parameter that changes this strain without changing the maximum stress. Substituting (8) and (9) to (7) we get: 


$$
\sigma_{0}=\frac{2 f_{c}\left(1-\alpha_{0}\right)}{\varepsilon_{0 p}^{*}}\left(\sqrt{2 \varepsilon_{p} \varepsilon_{0 p}^{*}}-\varepsilon_{p}\right)+\alpha_{0} f_{c} .
$$

The hardening parameter responsible for the evolution of the yield surface takes the form:

$$
H=\frac{d \sigma_{0}}{d \varepsilon_{p}}=\frac{2 f_{c}\left(1-\alpha_{0}\right)}{\varepsilon_{0 p}^{*}}\left(\sqrt{\frac{\varepsilon_{0 p}^{*}}{2 \varepsilon_{p}}}-1\right) .
$$

As a consequence of the assumed hardening model, the current limit state condition has to be introduced. In order to satisfy the requirement described above, the concepts of the failure surfaces given by J. Podgórski and K. J. Willam et al. have been respectively modified to enable the isotropic hardening by the uniform increase in their volumes. A new parameter $\sigma_{0}$ has been introduced for these two surfaces. In the case of the failure surface given by J. Podgórski, the current limit state condition is adopted in the following form:

$$
F\left(\sigma_{o}, \tau_{o}, \varphi, \sigma_{0}\right)=\frac{I_{1}}{3 \sigma_{0}}-\kappa+\frac{C_{1} P(J) \sqrt{6 J_{2}}}{3 \sigma_{0}}+\frac{2 C_{2} f_{c} J_{2}}{3 \sigma_{0}^{2}}=0,
$$

while in the case of the failure surfaces of Willam et al., this condition takes the form:

$$
F\left(\xi, \rho, \theta, \sigma_{0}\right)=2 \frac{A_{f}^{2}}{\sigma_{0}^{2}} f_{c}^{2} J_{2}+\frac{m f_{c}}{\sigma_{0}}\left[B_{f} \sqrt{2 J_{2}} r(\theta, e)+\frac{1}{3} C_{f} I_{1} \sqrt{3}\right]-1=0,
$$

where $I_{1}$ - the first invariant of stress tensor and $J_{2}, J_{3}$ - the second and the third invariants of stress tensor deviator, respectively.

The plastic potential $G$ is adopted in the form of the Drucker-Prager conical surface

$$
G\left(\sigma, \sigma_{0}\right)=\frac{\operatorname{tg} \gamma}{\sqrt{6}} I_{1}+\sqrt{J_{2}}-k
$$

where $k$ - a constant of the potential surface and $\gamma-$ an angle of inclination of the cone side surface to the hydrostatic axis. The variation of the angle $\gamma$ is assumed to fit the experimental data resulting from the phenomenon of dilatancy as follows:

$$
\gamma\left(\sigma_{0}\right)=\frac{\gamma_{\max }-\gamma_{\min }}{\pi} \operatorname{arctg}\left\{\chi\left[\sigma_{0}-\left(1-\alpha_{0}\right) f_{c}\right]\right\}+\frac{\gamma_{\max }+\gamma_{\min }}{2} .
$$

If a sufficiently large parameter $\chi$ is used, then for the effective stresses $\sigma_{0}=\left(1-\alpha_{0}\right) f_{c}$ there occurs a sudden skip from the value of $\gamma \rightarrow \gamma_{\min }$ to the value of $\gamma \rightarrow \gamma_{\max }$. The proper selection of parameters $\gamma_{\min }$ and $\gamma_{\max }$ enables more accurate reflection of the experimental curves by the results obtained theoretically.

\section{Comparison of predictions and experimental results}

The comparison of the effects of the numerical analysis of both above-mentioned models with the results of the experimental studies of Kupfer et al. [1, 2] has been presented in Figures 3-7. There have been considered the cases of uniaxial and biaxial compression. The following gradation of principal stresses has been adopted: $\sigma_{1} \leq \sigma_{2} \leq \sigma_{3}$. The material data characterizing the studied plain concrete have been used as input to the program, i.e. it has been assumed that: $E_{0}=33 \mathrm{GPa}$, Poisson's ratio $v=0.195$, uniaxial compression strength $f_{c}$ $=32.4 \mathrm{MPa}$ and strength parameters $\alpha_{t}=0.1, \alpha_{c c}=1.16, \alpha_{0}=0.3, \chi=5000, \gamma_{\min }=0^{\circ}, \gamma_{\max }=$ 
$12^{\circ}$. In the case of the analysis for the failure surfaces given by Willam et al. $[5,11]$, the assumed parameter $e$ is equal to 0.53 .

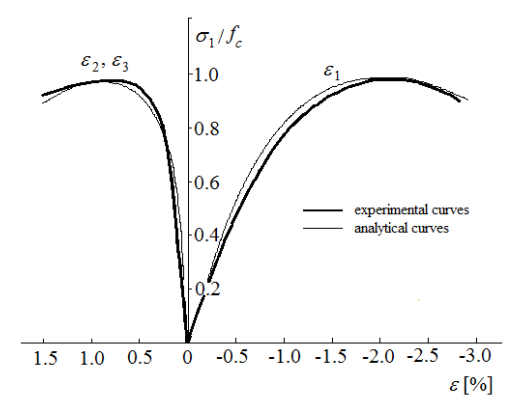

a)

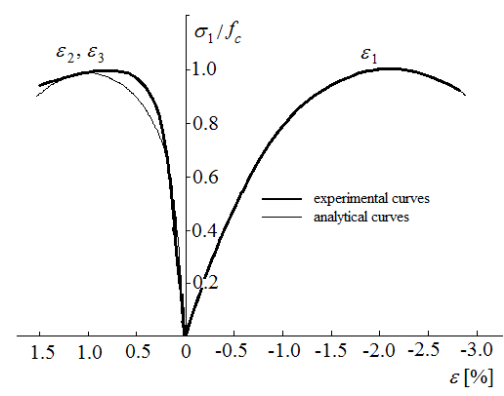

b)

Fig. 3. Comparison of the analytical and test results [2] for uniaxial compression: $\sigma_{2} / \sigma_{1}=0.0 /-1.0$ : a) Comparison for JP [9] failure surface, b) Comparison for W\&W [5, 11] failure surface.

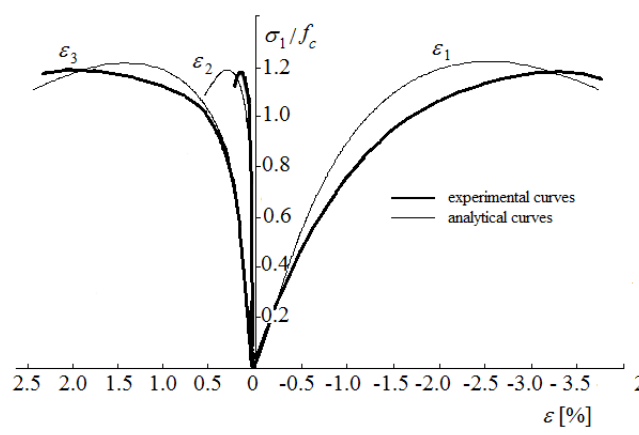

a)

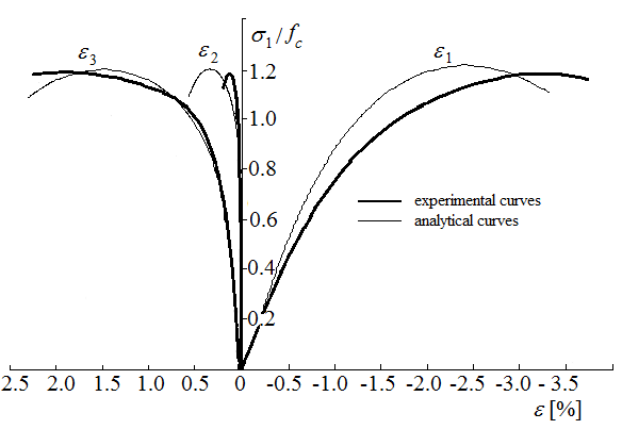

b)

Fig. 4. Comparison of the analytical and test results [2] for biaxial compression: $\sigma_{2} / \sigma_{1}=-0.226$ /-1.0: (a) Comparison for JP [9] failure surface, (b) Comparison for W\&W [5, 11] failure surface.
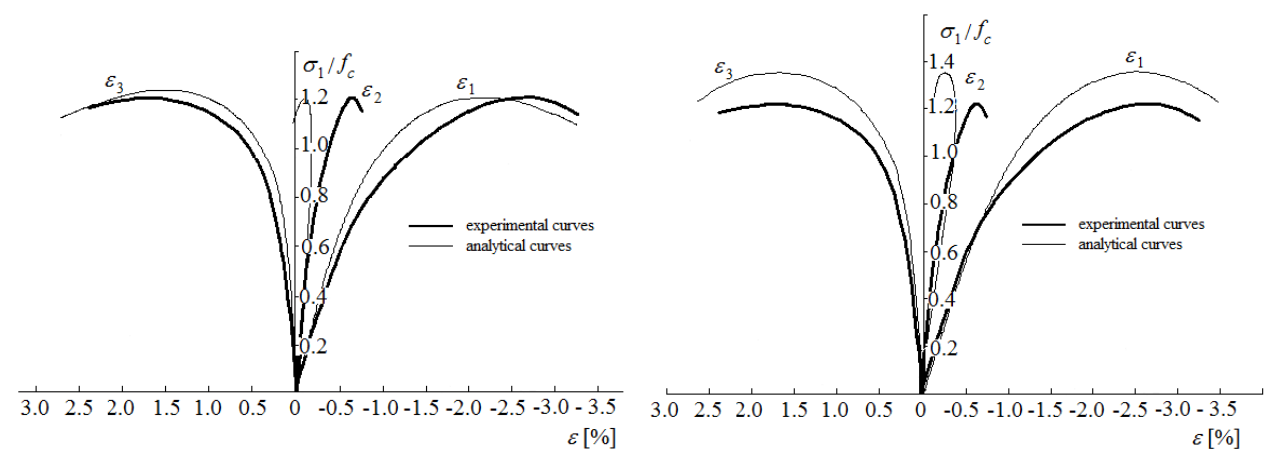

a) b)

Fig. 5. Comparison of the analytical and test results [2] for biaxial compression: $\sigma_{2} / \sigma_{1}=-$ 0.525/-1.0: (a) Comparison for JP [9] failure surface, (b) Comparison for W\&W [5, 11] failure surface. 

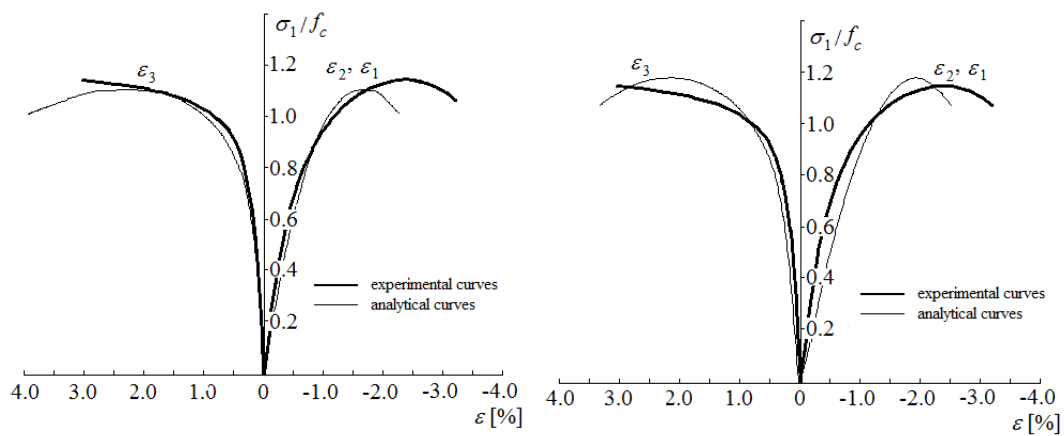

a)

b)

Fig. 6. Comparison of the analytical and test results [2] for biaxial compression: $\sigma 2 / \sigma_{1}=-1.0 /-1.0$ : (a) Comparison for JP [9] failure surface, (b) Comparison for $\mathrm{W} \& \mathrm{~W}[5,11]$ failure surface.

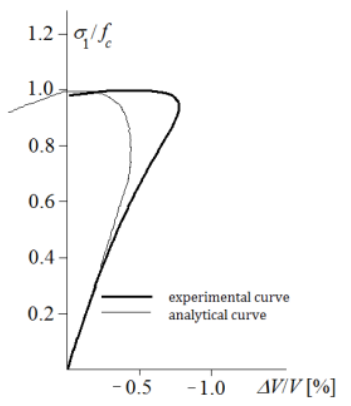

a)

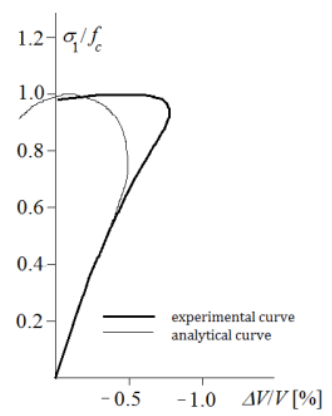

b)

Fig. 7. Plots of the volumetric changes due to the analytical and test results for uniaxial compression: (a) Comparison for JP [9] failure surface, (b) Comparison for W\&W [5, 11] failure surface.

\section{Summary and conclusions}

The present study shows the proposal for two elastic-plastic constitutive models for the multiaxial behaviour of concrete with the nonlinear work-hardening rule under short-term loading. The results of the numerical calculations for the plane stress state have been compared with the results of the experimental studies. Based on the results of such a comparative analysis, the following conclusions are drawn:

- The figures from Figure 3 to Figure 7 show a comparison of plots of stresses obtained as a result of the numerical analysis with experimental curves. Small stress increments had to be assumed in order to obtain the results with the required accuracy. From the numerical tests it follows that the load increment should not exceed 0.2 MPa and this enables a stable tracing of the descending branches of the stress plots.

- Despite the differences in the proposed actual surfaces, in both cases the best compatibility of the analytical and test results has been observed for the uniaxial compression. This is precisely the result of the utilisation of this state in the hardening model, while the biggest discrepancy has been observed for the volumetric changes. It is visible that for the plane stress state this compliance is somewhat lower than for the uniaxial stress state, however the differences are acceptable.

- Considering the comparisons of the numerical results with the experimental curves, the model utilizing the commonly recognized surface of Willam et al. $[5,11]$ coincides entirely with the experimental curve for the uniaxial stress. The outputs obtained based on both 
surfaces are largely comparable, therefore the selection of the best model is guided mainly by the behaviour of concrete in accordance with the test results under consideration and the simplicity of the formulation.

- To obtain more accurate comparisons of the proposed models of concrete for the cases of the three-dimensional stress state, it is possible to introduce the dependency of the hardening rule on the confining pressure acting on the side surfaces of cylindrical samples (see Lewinski and Dudziak [22]). In particular cases, it is also possible to seek a more proper control of the plastic potential evolution in order to reflect the phenomenon of dilatancy more accurately.

- The models presented herein can be used only for the first few load cycles. They can also be used as a starting point to devise a more sophisticated model [19].

\section{References}

1. H.B. Kupfer, K.H. Gerstle, J. Eng. Mech. Div., Proc. ASCE, 99 (EM4), 853 (1973)

2. H.B. Kupfer, H.K. Hilsdorf, H. Rüsch, ACI Journal, 66 (8), 656 (1969)

3. G. Schickert, H. Winkler, Deutscher Ausschuss für Stahlbeton, 277 (1977)

4. P.M. Zisopoulos, M.D. Kotsovos, M.N. Pavlovic, Cement \& Concrete Res., 30 (1), 153 (2000)

5. K.J. Willam, E.P. Wranke, IABSE Seminar on concrete structures subjected to triaxial stresses, III-1, Bergamo, Italy, May (1974)

6. A.C.T. Chen, W.F. Chen, J. Eng. Mech. Div., Proc. ASCE, 101 (EM4), 465 (1975)

7. N.S. Ottosen, J. Eng. Mech. Div., Proc. ASCE, 103 (EM4), 527 (1977)

8. P.V. Lade, J. Eng. Mech. Div., Proc. ASCE, 108 (EM5), 850 (1982)

9. J. Podgórski, Arch. Mech., 36 (3), 323 (1984)

10. Y. Ohtani, W.F. Chen, Computers \& Structures, 33 (4), 1047 (1989)

11. Ph. Menétrey, K.J. Willam, ACI Structural Journal, 92 (3), 311 (1995)

12. M. Klisiński, Degradation and Plastic Deformation of Concrete (in Polish), IFTR Reports, 38 (Warszawska Drukarnia Naukowa, Warszawa, 1984)

13. Lewiński P. M., $2^{\text {nd }}$ Int. Sci. AMCM Conference, Łódź, Poland, June, 405 (1996)

14. S. Jemioło, A. Szwed, $2^{\text {nd }}$ Int. Sci. AMCM Conference, Łódź, Poland, June, 165 (1996)

15. S. Jemioło, P.M. Lewiński, A. Szwed, EURO-C International Conference on Computer Modelling of Concrete Structures, Innsbruck, 1, 103 (1994)

16. P.M. Lewiński, M. Zygowska, $7^{\text {th }}$ AMCM Int. Conf.: Analytical Models and New Concepts in Concrete and Masonry Structures, Kraków, Poland, June, 345 (2011)

17. W. F. Chen, SM Archives, 13 (1), 1 (1988)

18. W. F. Chen, Plasticity in Reinforced Concrete, (J. Ross Publ., Fort Lauderdale, USA, 2007)

19. S. Tahaei Yaghoubi, R. Kouhia, J. Hartikainen, K. Kolari, Rakenteiden Mekaniikka (J. Struct. Mech.), 47 (2), 50 (2014)

20. J. Podgórski, Mechanics and Control, 30 (4), 229 (2011)

21. J. Podgórski, CMM 2013 - Computer Methods in Mechanics, Poznań, Poland, August, MS15-9 \& CD ROM (2013)

22. P.M. Lewiński, S. Dudziak, XXVI R-S-P Seminar: Theoretical Foundation of Civil Engineering, MATEC Web of Conferences 117, Warsaw, Poland, August (2017) 